\title{
First detection of Lyman continuum escape from a local starburst galaxy
}

\section{Observations of the luminous blue compact galaxy Haro 11 with the Far Ultraviolet Spectroscopic Explorer (FUSE) ${ }^{\star}$}

\author{
N. Bergvall ${ }^{1}$, E. Zackrisson ${ }^{1}$, B.-G. Andersson ${ }^{2}$, D. Arnberg ${ }^{1}$, J. Masegosa ${ }^{3}$, and G. Östlin ${ }^{4}$
}

1 Dept. of Astronomy and Space Physics, Box 515, 75120 Uppsala, Sweden

e-mail: [nils.bergvall; erik.zackrisson] @astro.uu.se

2 Department of Physics and Astronomy, Johns Hopkins University, 3400 North Charles Street, Baltimore, MD 21218, USA e-mail: bg@pha.jhu.edu

3 Instituto de Astrofisica de Andalucia, Granada, Spain e-mail: pepa@iaa.es

${ }^{4}$ Stockholm Observatory, SCFAB, 10691 Stockholm, Sweden e-mail: ostlin@astro.su.se

Received 7 July 2005 / Accepted 2 November 2005

\section{ABSTRACT}

Context. The dominating reionization source in the young universe has not yet been identified. Possible candidates include metal poor dwarf galaxies with starburst properties.

Aims. We selected an extreme starburst dwarf, the Blue Compact Galaxy Haro 11, with the aim of determining the Lyman continuum escape fraction from UV spectroscopy.

Methods. Spectra of Haro 11 were obtained with the Far Ultraviolet Spectroscopic Explorer (FUSE). A weak signal shortwards of the Lyman break is identified as Lyman continuum (LyC) emission escaping from the ongoing starburst. From profile fitting to weak metal lines we derive column densities of the low ionization species. Adopting a metallicity typical of the H II regions of Haro 11, these data correspond to a hydrogen column density of $\sim 10^{19} \mathrm{~cm}^{-2}$. This relatively high value indicates that most of the LyC photons escape through transparent holes in the interstellar medium. We then use spectral evolutionary models to constrain the escape fraction of the produced LyC photons.

Results. Assuming a normal Salpeter initial mass function we obtain a Lyman continuum escape fraction of $f_{\text {esc }} \sim 4-10 \%$. We argue that in a hierarchical galaxy formation scenario, the upper limit we derive for the escape rate allows for a substantial contribution to cosmic reionization by starburst dwarf galaxies at high redshifts.

Key words. galaxies: evolution - galaxies: formation - galaxies: starburst - galaxies: dwarfs - ultraviolet: galaxies cosmology: diffuse radiation

\section{Introduction}

According to WMAP data, the epoch of reionization started at a redshift of $z \approx 20$ (Kogut et al. 2003). Observations of the high opacity in the Lyman continuum (hereafter LyC) emission of galaxies (Becker et al. 2001; Fan et al. 2002) and the results from the recent studies of the intergalactic medium (IGM) around SDSS quasars (e.g. Mesinger \& Haiman 2004) indicate that the final stage of the epoch of reionization may have occurred at redshifts $z \sim 6-7$. There is still no consensus

* Based on observations made with the NASA-CNES-CSA Far Ultraviolet Spectroscopic Explorer. FUSE is operated for NASA by the Johns Hopkins University under NASA contract NAS-32985. about what could have been the major ionization sources of the IGM during the reionization epoch - stars, normal galaxies, AGNs or some exotic mechanism (Yan \& Windhorst 2004; Meiksin \& White 2003). The rapid decline of the space density of quasars at redshifts $z>3$ (Madau et al. 1999; Fan et al. 2001) makes it likely that their contribution to the LyC production rate required for the reionization is insignificant, although it has been proposed that miniquasars fed by intermediate-mass black holes may have been important (Madau et al. 2004).

At lower redshifts, observations of a sample of Lymanbreak galaxies at $z \sim 3.4$ (Steidel et al. 2001) seem to show that galaxies could account for a significant fraction of the photon flux required for reionization. The observations indicate a 
contribution of LyC photons a factor of $\sim 5$ higher than for quasars at the same epoch. These results have however not been confirmed by other similar studies. On the contrary, it has been argued (Fernández-Soto et al. 2003) that a maximum of $4 \%$ of the LyC flux could escape from luminous galaxies at redshifts $1.9<z<3.5$. This is also close to the upper limit of the escape fraction of low redshift galaxies (Leitherer et al. 1995; Hurwitz et al. 1997; Deharveng et al. 2001; Heckman et al. 2001). The lack of a strong trend with redshift indicates that galaxies at higher redshifts also have low escape fractions, insufficient to keep the intergalactic gas ionized. Alternative ionization sources that have been discussed are massive pregalactic Pop III stars and young globular clusters. Most probably the reionization is ruled by different sources at different redshifts. It is therefore important to establish observational constraints on the LyC fluxes from the various candidates at different epochs.

Our information about the galaxy luminosity/mass function at the epoch of reionization is very meager, and it is not at all clear what mass scale would dominate the LyC photon production rate. In a galaxy formation scenario where mergers are of major importance one would expect starburst dwarf galaxies to be frequent among the first generation of galaxies. Indeed the number density of so called Luminous Compact Blue Galaxies (LCBGs) appears to increase with redshift (Lilly et al. 1998; Mallén-Ornelas et al. 1999). It is therefore important to investigate if this type of galaxy could have been an important ionization source at high redshift. Dwarf galaxies are however too faint to be observed at high redshifts. We are therefore dependent on theoretical models and observations of nearby galaxies that can be used as templates of the first generation of dwarf galaxies. Recent models do not give much hope for Lyman escape from galaxies of masses larger than $10^{7}$ solar masses (Kitayama et al. 2004) but these predictions have to be tested observationally.

We have searched for local dwarfs with properties similar to young dwarf galaxies, that could be used as test objects. A few such studies have already been carried out but only provided upper limits to the escape fraction (e.g., Giallongo et al. 1997; Devriendt et al. 1998; Shull et al. 1999). However, predicted equivalent widths of the Balmer emission lines in starbursts are in some cases larger than observed (e.g. Bergvall 1985; Bresolin et al. 1999; Mas-Hesse \& Kunth 1999; Moy et al. 2001; Stasinska et al. 2001). Several possible explanations to this were discussed by Mas-Hesse \& Kunth (1999). One possibility is that the most massive stars remain hidden in their parental clouds until most of the fuel has been consumed. This would mimic a truncation of the IMF at high masses. Mas-Hesse and Kunth also discussed the possibility that a large fraction of the ionizing photons are absorbed by dust in the $\mathrm{H}$ II regions. To obtain agreement with the observations, a large amount of the photons, $\sim 60 \%$ would have to be absorbed. This possibility has recently been investigated by Hirashita et al. (2003) where the escape fraction of the LyC photons from the $\mathrm{H}$ II region itself is estimated from model comparisons. Based on input data from a sample of star forming galaxies they find that, in the mean about $40 \%$ of the photons are trapped, which is less than what would be needed according to Mas-Hesse and Kunth. But the scatter is high so in some cases this certainly would be a possibility. Hirashita et al. seem to find that the escape fraction is higher in galaxies with properties similar to Haro 11 (e.g. the BCG ESO 338-IG04). Therefore an alternative, or complementary, explanation for the low flux of the recombination lines, may be that a significant fraction of the LyC photons is in fact escaping from the galaxy. This is the possibility that we study in this paper.

$\mathrm{H}$ II regions in nearby galaxies appear to leak $40-50 \%$ of the LyC photons produced by the hot stars into the diffuse interstellar medium (Ferguson et al. 1996; Oey et al. 1997). Although it cannot be completely excluded that the gas may be ionized by single $\mathrm{O}$ stars located in situ, a comparison of the observed emission line strengths with theoretical models (Iglesias-Páramo \& Muñoz-Tuñón 2002; Wood \& Mathis 2004) give support to the leakage interpretation. An even more extreme case of a density bounded H II region around a massive star cluster was reported by Leitherer et al. (1996). They estimate that about $75 \%$ of the $\mathrm{LyC}$ radiation is leaking into the diffuse ambient medium. Depending on the distribution of the gas, the location where these photons are absorbed may be very different from case to case. In some situations the diffuse ionized gas may be much more extended than the $\mathrm{H}$ II region producing the photons and will be ignored by the observer who consequently will underestimate the star formation rate.

The other alternative for the discrepancy between theory and observations of the Balmer emission line strengths is that the dust is unevenly distributed across the starburst region so that the younger regions are more reddened than the older ones (Calzetti et al. 1994). This could reduce the equivalent width of $\mathrm{H} \alpha$ with up to a factor of $\approx 2$. The only way to really distinguish between a global leakage into to intergalactic medium and the other two alternatives is to search for leaking photons shortward of the Lyman limit.

In previous studies of starburst dwarfs (Bergvall 1985; Bergvall \& Östlin 2002; Östlin et al. 1999, 2001) we have focused on a few luminous $\left(-19>M_{B}>-21\right)$ metal poor blue compact galaxies (BCGs). They have properties similar to the LBGs at intermediate redshifts (e.g. Guzman et al. 2003). Here we report on such a study using FUSE, the Far Ultraviolet Spectroscopic Explorer.

\subsection{The target galaxy}

The target galaxy of our choice is Haro 11 (=ESO 350-IG38) at $\alpha_{2000}=00^{\mathrm{h}} 36^{\mathrm{m}} 52.5^{\mathrm{s}}, \delta_{2000}=-33^{\circ} 49^{\prime} 49^{\prime \prime}$. From the emissionline spectrum we derive a heliocentric radial velocity of $v_{0}=6180 \mathrm{~km} \mathrm{~s}^{-1}$, corresponding to a distance of $86 \mathrm{Mpc}$, assuming a Hubble constant of $72 \mathrm{~km} \mathrm{~s}^{-1} \mathrm{Mpc}^{-1}$. We have previously studied this galaxy over a wide frequency range, from UV to radio (Bergvall \& Olofsson 1986; Bergvall et al. 2000, 2002; Östlin et al. 1999, 2001). It has unique properties that makes it one of the most relevant local objects for our purpose. The chemical abundances are low, the oxygen abundance being $\sim 20 \%$ solar (relative to the revised solar oxygen abundance by Asplund et al. 2004b).

An estimate of the typical hydrogen mass of a BCG can be obtained from the correlation between gas mass and major 
diameter (Gordon \& Gottesman 1981). Haro 11 has a major diameter of $11 \mathrm{kpc}$ at $\mu_{B}=25 \mathrm{mag} \operatorname{arcsec}^{-2}$ indicating that the $\mathrm{H}$ I mass should be approximately $2 \times 10^{9} \mathcal{M}_{\odot}$. Despite several efforts at VLA, Nancay and Parkes (all unpublished) we have not been able to detect $\mathrm{H}$ I in this galaxy. We estimate the upper limit to $\mathcal{M}(\mathrm{H} \mathrm{I}) \sim 10^{8} \mathcal{M}_{\odot}$. The extraordinary strong [C II] $\lambda 158 \mu$ line, observed with ISO (Bergvall et al. 2000), shows that a large part of the H I gas is located in photodissociation regions (PDRs). We have estimated the contribution from $\mathrm{H}_{2}$ and $\mathrm{H}$ II gas to between $10^{8}$ and $10^{9} \mathcal{M}_{\odot}$ each. This would leave a remarkably small fraction of the total gas mass in atomic form. A possible explanation is that the bulk of the gas in the halo that normally is in neutral state has become ionized by the starburst. Our $\mathrm{H} \alpha$ observations show that the main body is indeed surrounded by an extended halo of ionized gas. The conditions for $\mathrm{LyC}$ photon escape thus appear to be unusually favourable. It is interesting to note that the nearby BCG Pox 186, sometimes called "ultracompact", is also H I quiet (Begum \& Chengalur 2005).

HST GHRS UV spectroscopic observations of Haro 11 were carried out by Kunth et al. (2003) who found a quite complex absorption system with velocity components of Ly $\alpha$ in absorption on both sides of the emission line. Thus it appears that there are both outflows and infall of gas in the centre direction. From the absorption lines Kunth et al. estimate column densities and find values as high as $\log n_{\mathrm{H}} \sim 20 \mathrm{~cm}^{-2}$. The aperture used is small however ( 2 " circular diameter) and covers only the very central part of the galaxy. Our optical spectroscopy (Östlin et al. 2005) reveals velocity gradients of both gas and stars as large as $200 \mathrm{~km} \mathrm{~s}^{-1}$ across two of the bright condensations in the very centre, indicating an extremely high mass density in this region.

\section{Observations and reductions}

\subsection{IUE observations}

In 1984 we observed the galaxy in low dispersion mode with the SWP UV spectrograph of the IUE spacecraft. The aperture used was $10^{\prime \prime} \times 20^{\prime \prime}$. A low resolution spectrum is shown in Fig. 1. Below we will use the slope of the spectrum as an input to the derivation of the dust extinction.

We will also use the IUE spectrum to determine the C/O abundance ratio, based on the C III] $\lambda 1908$, and O III] $\lambda 1663$ lines. The strongest line, also representing the dominating ionization stage is C III] $\lambda 1908$ while the O III] line is nosier. Thus the error in the determination will be significant but it still will be useful as a check of the $\mathrm{C} / \mathrm{O}$ ratio determined from the FUSE absorption lines (see Sect. 3.1). The atomic collision strengths are determined from the electron temperature derived by Bergvall \& Östlin (2002) $T \approx 13400 \mathrm{~K}$. From the relative line intensities we then obtain $N\left(\mathrm{C}^{+}\right) / N\left(\mathrm{O}^{++}\right) \sim$ $0.3 \pm 0.1$. The error is dominated by the photon statistics and has been estimated from the noise in the region around the line. Assuming ionization correction factors $\operatorname{ICF}\left(\mathrm{C} / \mathrm{C}^{+}\right) \sim 1.4$ (Stasińska 1990) and $\mathrm{ICF}\left(\mathrm{O} / \mathrm{O}^{++}\right) \sim 1.5$ (Bergvall \& Östlin 2002), we then finally obtain $N(\mathrm{C}) / N(\mathrm{O})=0.46_{-0.10}^{+0.15}$.

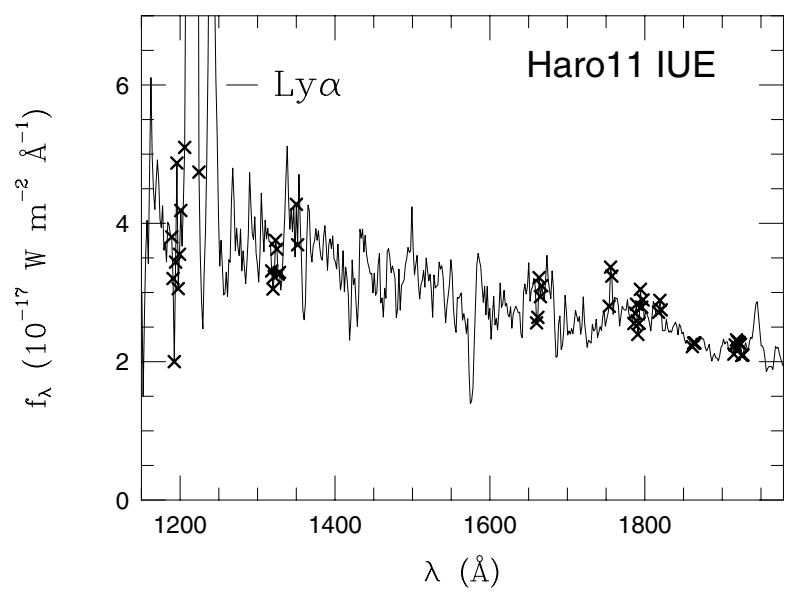

Fig. 1. Spectrum of Haro 11 obtained with the IUE spacecraft in the low resolution SWP mode. The aperture used was $10^{\prime \prime} \times 20^{\prime \prime}$. Bad pixels have been flagged with crosses.

\subsection{FUSE observations}

The data were obtained on Oct. 12, 2001, using the FUSE low resolution mode LWRS with an aperture of $30^{\prime \prime} \times 30^{\prime \prime}$. The total integration time on target was $16 \mathrm{ks} .12 \mathrm{ks}$ of the observations were carried out during orbital night. The data were reduced using CalFUSE v3.0, with default processing parameter for all spectral segments. As the background level in FUSE data is quite low and hence cannot be reliably measured in detail for short observations, the standard FUSE background correction is based on a set of template files which are scaled to averages over the whole detector in any given observation. These template files consist of coadded data from many, long observations of blank sky acquired throughout the mission. The FUSE detectors consist of microchannel plates with electronic pixels whose exact locations depend on the electrical field structure and read-out electronics of the detector (Sahnow et al. 2000). Artificial count enhancements occur at the edges of the detector due to non-uniformities in the field and have a very steep dependence on the distance from the edge. The exact mapping of the detector to location in the electronic detector-image depends on several factors including the total brightness of the detector illumination. Because of the steepness of this edge effect, minor mismatches in the target and background images can cause significant errors in the background subtraction (Sahnow 2005, private communication). As the $\mathrm{SiC}$ spectrum falls very close to the lower edge of detector $1 \mathrm{~B}$, any relative change in the strength of this edge effect compared to the rest of the background will cause problems in the background correction, particularly for weak sources such as Haro 11. To compensate for this effect, we reset the areas and relative weighting used to determine the scaling of the background template in the reduction of the SiC1B data. Without any further modifications the derived SiC1B spectrum then agrees well with the SiC2A spectrum. The regions of the lowest signal in both the SiC1B and the LiF1A segments were however significantly below the zero level, indicating that the proper background on the whole was fainter than the subtracted template background. After 


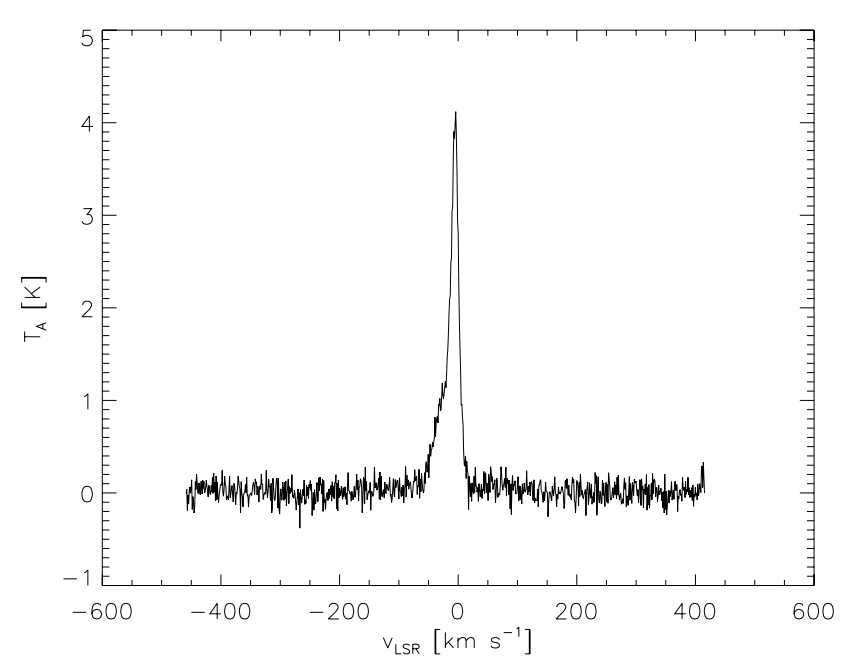

Fig. 2. The 21-m line in the direction of Haro 11 obtained from the Leiden/Dwingeloo survey (Burton \& Hartmann 1994). $V_{\text {LSR }}$ is the radial velocity relative to the local standard of rest and $T_{\mathrm{A}}$ is the antenna temperature.

extraction, calibration and combination of the individual segments, a constant was therefore added to the background to compensate for this. As we are more strongly constrained by low signal-to-noise than spectral resolution, we are not significantly affected by the slight difference in dispersion solution between segments, and the consequent lowering of resolution caused by this coaddition.

\section{Results}

Figure 3 shows the full spectrum with a few of the strongest absorption and emission lines indicated. Strong geocoronal Lyman emission lines are seen on top of the absorption spectrum. The narrow spike at $1168.7 \AA$ is a scattered HeI solar emission line. From the absorption lines we can define two velocity systems, one originating in the Milky Way and the other in Haro 11. Figure 2 shows the H I 21-cm line spectrum in the direction of Haro $11\left(l=328^{\circ}, b=-83^{\circ}\right)$, obtained from the Leiden/Dwingeloo survey (Burton \& Hartmann 1994). Our analysis shows that the spectrum in the velocity range $-450 \mathrm{~km} \mathrm{~s}^{-1}-400 \mathrm{~km} \mathrm{~s}^{-1}$ can be approximated with two components with small negative velocities relative to the LSR as shown in Table 1.

The spectral features identified to belong to the Milky Way ISM are indicated with hatched vertical lines in Fig. 3. These absorption lines have a linewidth of $F W H M \sim 30 \mathrm{~km} \mathrm{~s}^{-1}$ in agreement with the $F W H M$ given in Table 1 . The narrowness of the lines is important since it ensures that we can find the proper level of the Lyman continuum of Haro 11 between the positions of the galactic Lyman absorption lines. The spectrum of Haro 11 is rich in spectral features and dominated by absorption lines from $\mathrm{O}$ and $\mathrm{B}$ stars. Possible detections of molecular hydrogen lines in the Lyman series have also been indicated. The heliocentric systemic velocity of Haro 11, as defined from the optical H II emission (unpublished) and the stellar CaII triplet absorption lines, weighted from the UV mapping of the galaxy (Östlin et al. 2005), is $v=6180 \mathrm{~km} \mathrm{~s}^{-1}$. This velocity agrees with the position of the P V $\lambda 1118 \AA\left(\lambda_{\text {obs }}=1141 \AA\right)$ line. The $\mathrm{P} V$ line originates from hot dwarf stars on the main sequence. If these stars are metal-rich and massive they tend to show P Cygni profiles while at lower metallicities, as in our case, the feature is mainly photospheric and narrow and can be used as a systemic velocity fixpoint. Thus we have two independent derivations of the systemic velocity of the stellar population seen in the far-UV spectral region. Strong deviations from this value would indicate in- or outflows of gas.

The strongest absorption lines, like those of the Lyman series, are dark but not saturated as is normally the case for starburst dwarfs (e.g. Heckman et al. 2001). This in itself is an indication that the optical depth in the $\mathrm{LyC}$ is lower than normal for this type of galaxies. Many of the absorption lines originating in Haro 11 are broad and show P Cygni profiles, e.g. the O VI $\lambda \lambda 1032,1038$ (redshifted to $\lambda 1053,1058 \AA$ ) lines. Most of these lines are probably formed in the photospheres or in the winds of $\mathrm{O}$ and $\mathrm{B}$ stars but there may also be a contribution from more global outflows. After a rough correction for the stellar contribution (see next section), some of the strongest absorption features show asymmetries, indicating high-velocity outflows. This aspect will be briefly discussed below and more in detail in a forthcoming paper, focused on the absorption line spectrum.

In Fig. 4 we display the short wavelength part of the spectrum. This spectral region is covered by two detector segments, $1 \mathrm{~B}$ and $2 \mathrm{~A}$, producing two independent spectra $\mathrm{SiC} 1 \mathrm{~B}$ and SiC2A. Wavelengths below $917 \AA$ are only covered by segment 1B. This is also the detector segment with the smallest effective area of the two. Thus, in this region the signal is noisier than at longer wavelengths. What is particularly interesting from Fig. 4 is that below the Lyman limit of Haro 11 there clearly seems to be a weak but significant signal in the Lyman continuum. Shortwards of the Lyman limit of the local rest frame the signal diminishes gradually, as expected from the increasing density of the Lyman absorption lines of the local ISM. The emission is seen in the 2-dimensional display of the spectral region as a faint narrow noisy structure in both $\mathrm{SiC}$ channels. We therefore feel confident that the LyC excess is not due to calibration problems. The level of the signal however is not so well determined, mainly because of uncertainties in the calibration, a low signal and possibly influences of auroral emission lines and emission and absorption lines from the Milky Way which we will now discuss.

Haro 11 is at a galactic latitude of $-83 \mathrm{deg}$. and thus interstellar absorption is not a serious problem. From Schlegel et al. (1998) we obtain $A_{B}=0.049^{\mathrm{m}}$ corresponding to $A_{\mathrm{Ly}-\text { limit }} \sim$ $0.18^{\mathrm{m}}$ (Mathis 1990). The Lyman emission lines from the terrestrial airglow are indicated in Fig. 5. For comparison we show in Fig. 6 both the spectrum based on night-and-day data and the spectrum based on night data only. We can clearly identify the geocoronal Lyman emission lines. Shortwards of the Lyman lines, at much lower intensities, C I lines may weakly influence the counts (Feldman et al. 2001). In the following discussion we will use the $900 \AA$ rest frame flux density of Haro 11 as a reference of the LyC level. This choice allows comparisons with previous studies of other galaxies (e.g. 


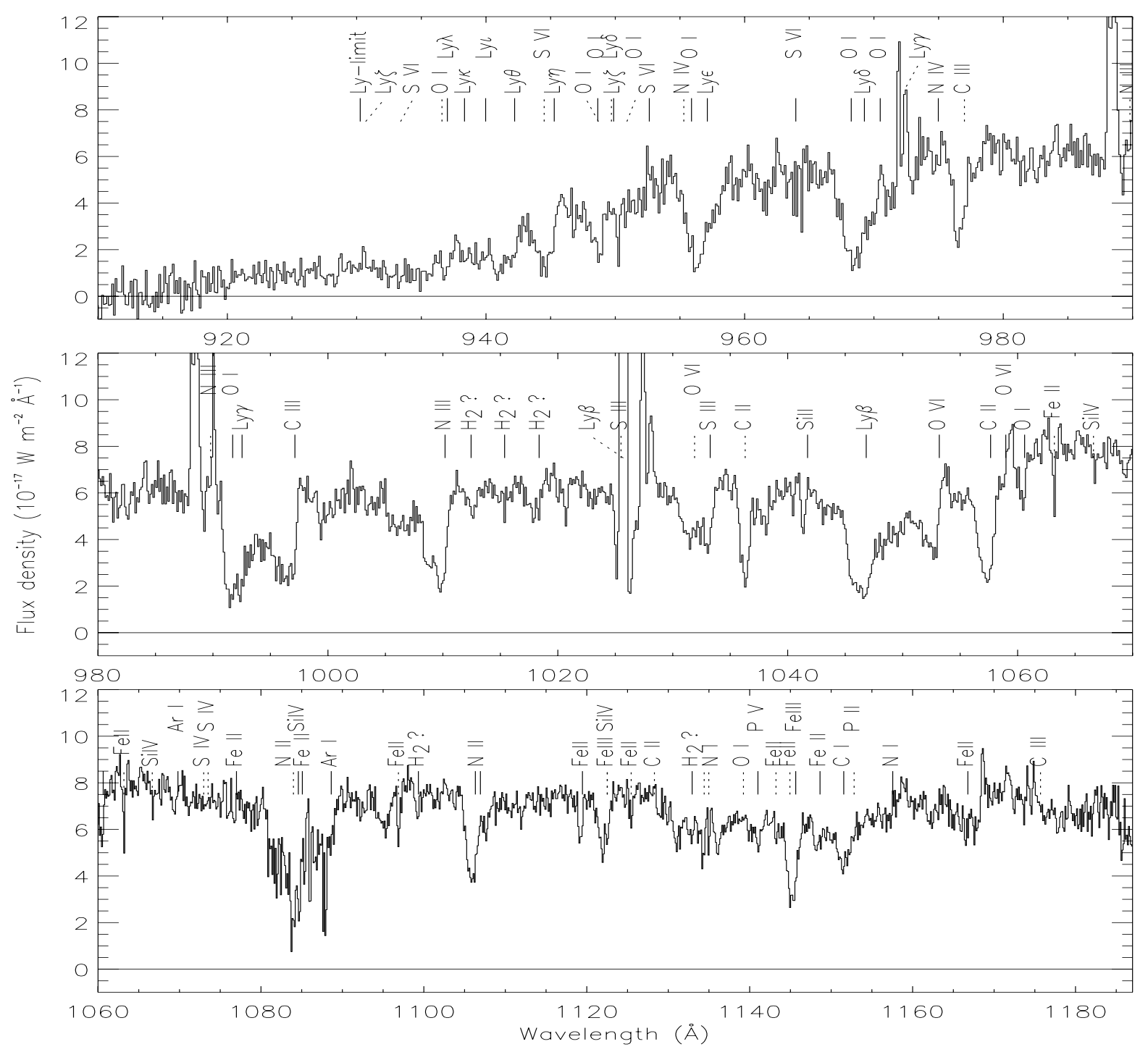

Fig. 3. FUSE spectrum of Haro11. The major absorption features have been indicated with solid lines (Haro 11 optical rest frame) and hatched lines (local frame). The spectrum also shows strong geocoronal Lyman emission lines and scattered solar HeI at $\lambda 1169 \AA$. The data have been rebinned with a boxcar of size $0.26 \AA$.

Leitherer et al. 1995; Deharveng et al. 2001; Inoue et al. 2002). In the local frame this part of the spectrum has been redshifted to $\lambda=920 \AA$. To beat down the noise in the spectrum we determine the LyC level over the line-free regions in the interval 920-925 $\AA$, since the LyC signal is not expected to vary significantly over such a short interval. After correcting for galactic extinction, we obtain $f_{900}=1.1 \pm 0.1 \times 10^{-17} \mathrm{~W} \mathrm{~m}^{-2} \AA^{-1}$. The uncertainty due to the complexity in the determination of the background is probably significantly larger than the formal error. In the worst case it could amount to of the order of $\sim 50 \%$ of the signal.

\subsection{Spatial distribution of the absorbers}

We mentioned in the introduction that Haro 11 appears to be devoid of neutral hydrogen. This could indicate that the LyC photons are leaking out from a truncated Strömgren sphere, i.e. that the emission is "density bounded". The
Table 1. Components of the local H I spectrum in the direction of Haro 11. The columns show the central velocity relative to the local standard of rest, peak antenna temperature, full width at half maximum and the derived column density.

\begin{tabular}{llll}
\hline \hline $\begin{array}{l}V(\text { centre }) \\
\mathrm{km} \mathrm{s}^{-1}\end{array}$ & $\begin{array}{l}T \text { (peak) } \\
\mathrm{K}\end{array}$ & $\begin{array}{l}F W H M \\
\mathrm{~km} \mathrm{~s}^{-1}\end{array}$ & $\begin{array}{l}\text { cm } \\
\mathrm{cm}^{-2}\end{array}$ \\
\hline-5.1 & 3.1 & 13.4 & $8.1 \times 10^{19}$ \\
-19.8 & 1.5 & 42.6 & $9.5 \times 10^{19}$ \\
\hline
\end{tabular}

hydrogen ionization cross section at the Lyman limit is $\approx 8 \times$ $10^{-18} \mathrm{~cm}^{2}$. This means that the LyC is essentially black at column densities of a few times $10^{17} \mathrm{~cm}^{-2}$, if the neutral gas is diffusely distributed. As discussed in Sect. 1.1 we have derived an upper limit of the mass of neutral hydrogen in Haro 11 of $10^{8} \mathcal{M}_{\odot}$. We can use this number to estimate the maximum column density of neutral hydrogen under the assumption that the 


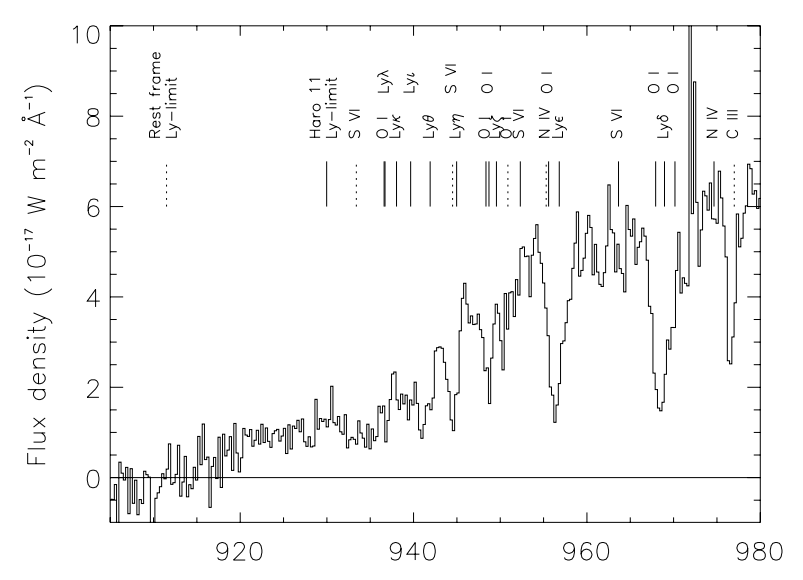

Fig. 4. Part of spectrum showing the Lyman continuum signal. The Lyman limit in the local frame (hatched lines) and in the frame of Haro 11 (solid lines) have been indicated.

$\mathrm{HI}$ has the same spatial distribution as the stars in Haro 11. The stellar scalelength is $h_{V} \sim 2.5 \mathrm{kpc}$ (Bergvall \& Östlin 2002). This gives an $\mathrm{H} \mathrm{I}$ column density in the direction of the centre of $N(\mathrm{H} \mathrm{I})=5 \times 10^{19} \mathrm{~cm}^{-2}$ which can be regarded as an upper limit, considerably larger than the limiting column density for Lyman leakage. In this simple model H I would become transparent at $4-5$ scalelengths (i.e. $\sim 10 \mathrm{kpc}$ ) from the centre, where the host galaxy component starts to dominate. But we know that the neutral gas is clumped so we will adapt the simplest alternative to the homogeneous model - the "picketfence" model. Here it is assumed that the LyC radiation is leaking out through holes in the ambient gas. In principle we can carry out profile fitting to the Lyman lines in absorption to derive the $\mathrm{H}$ I column densities. These lines are however not very useful since there is a mixed contribution from OB star photospheres, ISM absorption and line emission from the H II regions. We can make an estimate of the influence of stellar features from models of spectra of young stellar populations by Robert et al. (2003). These models are based on observed stellar spectra over a range of metallicities. The metal-poor models are valid for ages below $15 \mathrm{Myr}$. We interpolated the model spectra to a metallicity in agreement with what we obtained from the optical spectra, i.e. an oxygen abundance of $\sim 18 \%$ solar (based on the solar oxygen abundance derived by Asplund et al. 2004b). We also assumed a Salpeter initial mass function (IMF) and a constant star formation rate (SFR). Figure 7 shows the best fit to these models, a burst of the highest age in the library, 14 Myr. The optical spectra of the central burst show strong W-R features and, as we mention above, P Cygni profiles are seen in the FUSE spectrum. These do not fit to the model spectrum shown in Fig. 7. A better fit can be obtained if we assume an increasing SFR with the UV emission dominated by massive stars of an age of a few Myr. Modifications of the standard IMF can further improve the fit. There may also be a significant contribution to the P Cygni component from the supernova generated superwind. However, these issues are not of main importance here.

The influence of young stars in the spectrum is strong. The presence of O VI and its P Cygni profile indicates the presence

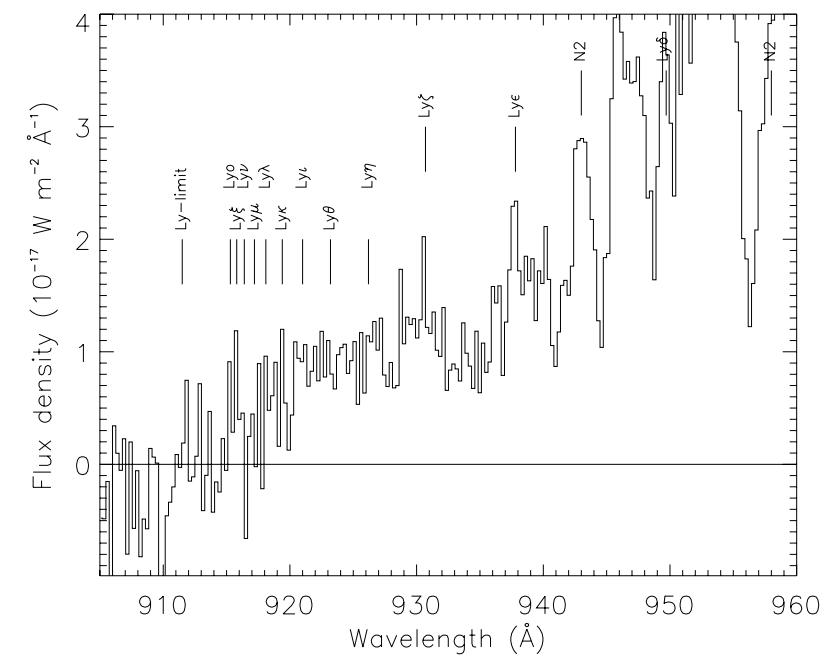

Fig. 5. Airglow lines and the rest frame Lyman series.

of O supergiants (e.g. González Delgado et al. 1997). Thus the burst must be active or stopped not more than 3-4 Myr ago. For a continuous SFR we do not expect the spectrum to change much over the expected maximum duration of a typical burst, $\sim 10^{8}$ yr and therefore the comparison in Fig. 7 should be qualitatively correct. The model spectrum has only partly been corrected for interstellar absorption but the influence on the $\operatorname{Ly} \beta$ line is marginal so the line profile should be entirely dominated by the stellar photospheric contribution. This is confirmed also from a comparison with predicted $\mathrm{Ly} \beta$ absorption line strengths in models of O star spectra (González Delgado et al. 1997). Thus it is obvious that these features have a strong influence and thus the Lyman lines cannot be used to derive the column density without a more careful correction for the influence of the stellar features. However, if we can derive column densities of low ionization stages of heavy elements with known abundances relative to hydrogen we can obtain a stricter constraint on the H I column density since the atoms and ions in these stages to a first approximation should coexist with the neutral hydrogen.

We will use a few rather well defined and isolated low ionization lines, C II $\lambda 1036$, O I $\lambda 1039$, SiII $\lambda 1021$, ArI $\lambda 1048$, FeII $\lambda 1097$ and $\lambda 1125$. We cannot exclude the fact that there may be a weak contribution to some of these lines from the stellar photospheres but the bulk of the absorption should originate in the ISM. The ionization potentials of these lines are slightly higher than 1 Ryd. It is known from studies of the local interstellar medium that elements with ionization potentials below that of hydrogen and the ionization potential of the second ionization stage above, exist predominantly in the singly ionized stage where hydrogen is neutral. It is therefore possible to use also these lines to obtain good estimates of $N(\mathrm{HI})$ under certain conditions. The lines of the neutral atoms $\mathrm{O}$ and $\mathrm{Ar}$ can directly be used to derive column densities if the atoms are effectively shielded from ionizing photons by the neutral hydrogen. In regions where H I is partially ionized, argon may be more fully ionized as a consequence of it high cross section to photon absorption. If so, we would underestimate the column 


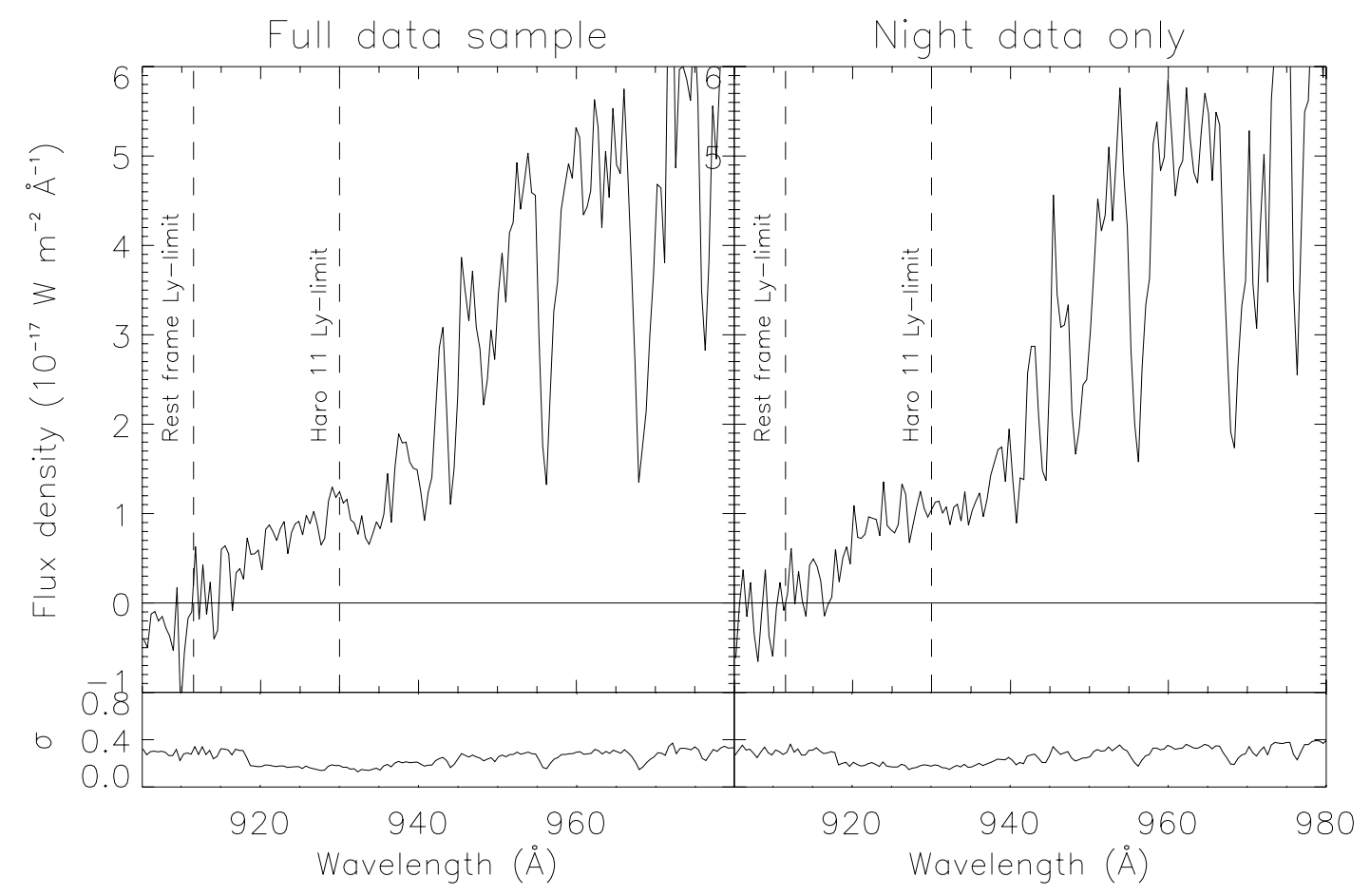

Fig. 6. Comparison between night and day data. The lower panel shows the $1 \sigma$ noise level, corrected to the binsize used in the plot.

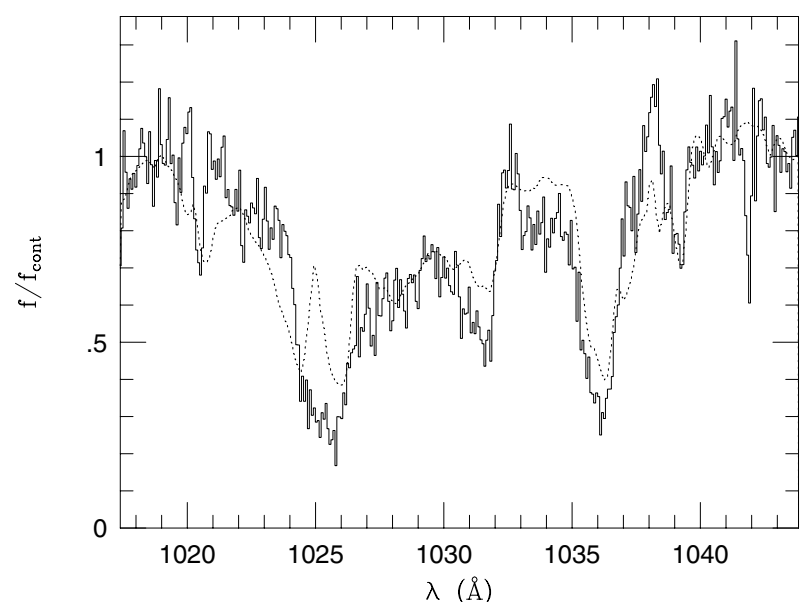

Fig. 7. The FUSE spectrum of Haro 11 (solid line) along with the best fitting stellar spectrum (dotted line) from Robert et al. (2003). The wavelength scale has been corrected to the rest frame of Haro11, assuming a systemic heliocentric velocity of $6180 \mathrm{~km} \mathrm{~s}^{-1}$. The model spectrum is based on a Salpeter IMF, a stellar mass range of 1-120 $\mathcal{M}_{\odot}$, a metallicity of $18 \%$ solar and a continuous SFR. The age is $14 \mathrm{Myr}$.

density based on Ar I. This seems to be confirmed from the results in Table 2. Oxygen behaves differently since the ionization fraction is coupled to that of hydrogen via resonant chargeexchange reactions (Jenkins et al. 2000).

The optical depth of a specific species is given by:

$\tau(\lambda)=\frac{\pi e^{2}}{m_{e} c^{2}} f \lambda^{2} N(\lambda)$
Table 2. Column densities of low ionization species.

\begin{tabular}{llll}
\hline \hline Species & $\lambda$ & $\log N$ & $\sigma_{\log N}$ \\
\hline $\mathrm{O}$ & 1039.2 & 15.48 & 0.02 \\
$\mathrm{C}$ & 1036.3 & 15.19 & 0.02 \\
$\mathrm{Si}$ & 1020.7 & 15.10 & 0.03 \\
$\mathrm{Ar}$ & 1048.2 & 13.67 & 0.03 \\
$\mathrm{Fe}$ & 1096.9 & 14.80 & 0.08 \\
& 1125.4 & 14.59 & 0.13 \\
\hline
\end{tabular}

where $f$ is the oscillator strength. From this we can derive the column density in $\mathrm{cm}^{-2}$ :

$N=\int N(v) \mathrm{d} v=\frac{3.768 \times 10^{14}}{f \lambda} \int \tau(v) \mathrm{d} v$

where $\lambda$, the wavelength of the line, is given in $\AA$ and $v$ is the velocity in $\mathrm{km} \mathrm{s}^{-1}$.

We derived the column densities using the Owens software obtained from the French FUSE team. The line minimum corresponds to the optically derived radial velocity. The typical virial velocity of BCGs of this luminosity is $<100 \mathrm{~km} \mathrm{~s}^{-1}$. The width of the absorption lines are much larger than this. This is probably due to outflows from the starburst and to a lesser extent also to infall of neutral gas. We have to adopt the fit of the line profiles to this condition and the programme we use conveniently allows one to fit lines belonging to several velocity components simultaneously. Our approach was to test a number of situations in which we assumed the cloud ensemble to consist of between 3 and 9 different velocity systems, in all cases covering the whole width of the lines. In the cases where the number of components were low this was 


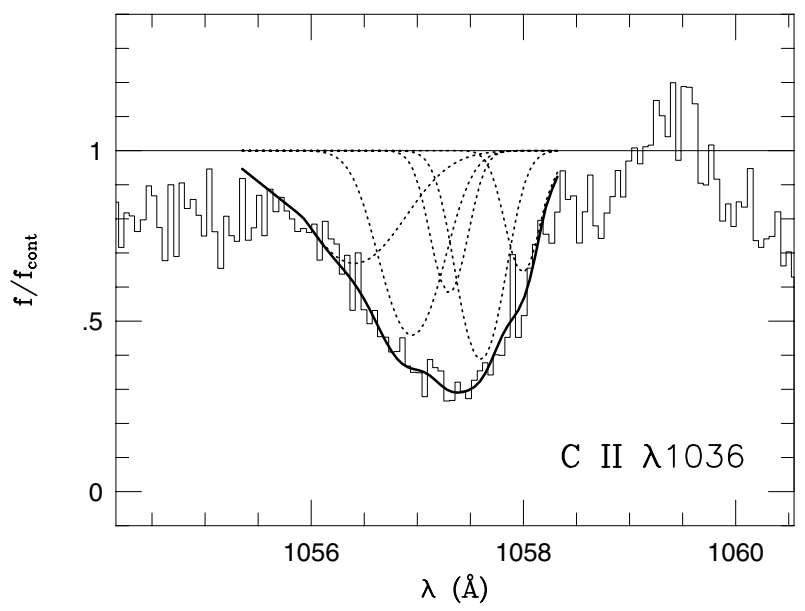

Fig. 8. The best free fit to the observed C II $\lambda 1036$ line based on 5 velocity components with different Doppler widths and component separations. The strongest component is centered on the systemic velocity as derived from the optical data.

compensated by the software by increasing the Doppler broadening parameter, $b$. The $b$ parameter was allowed to vary between 30 and $180 \mathrm{~km} \mathrm{~s}^{-1}$. FUSE has a resolution of $\sim 20 \mathrm{~km} \mathrm{~s}^{-1}$ and one might worry that there could be a significant number of unresolved saturated components with low $b$ parameters in the sightline. From FUSE curve-of-growth of neutral and low ionization absorption line systems in the Magellanic bridge one obtains $b$ parameters in the range $15-20 \mathrm{~km} \mathrm{~s}^{-1}$ (Sembach et al. 1979; Lehner 2002). The $\mathrm{H}_{2}$ lines give smaller $b$ values, indicating a stronger confinement and lower turbulence of these clouds. We do not expect the conditions in Haro 11 to be more quiet than in the Magellanic bridge and therefore we should be safe with a lower value of the $b$ parameter of the low ionization lines of $30 \mathrm{~km} \mathrm{~s}^{-1}$. In the example we discuss below, however, we allow the parameter to vary freely, utilizing the full resolution of FUSE. In one setup of parameters we fixed the relative velocity differences between the different components to the same value, so that the full width of the lines was covered. We also tested fits where both the velocity differences and the line widths were allowed to vary freely. In all cases we assumed a gas temperature of $300 \mathrm{~K}$. The absorption lines from all atoms and ions under study were included simultaneously in the fits. The optimum fits were found to contain between 5-7 components. Ideally one would expect the relative strengths of the components to be the same for each element. As the components get weaker and noiser however, the results become more and more uncertain. Thus while the central 3-4 components show a good agreement, the fainter components may deviate significantly from one species to the other. The goodness of fit (lowest total $\chi^{2}$ residual/numbers of degrees of freedom) showed small variations between fits to different numbers of subcomponents. As an example, one of best results for the carbon line is shown in Fig. 8.

From Table 2 we obtain an oxygen to carbon ratio of $N(\mathrm{C}) / N(\mathrm{O}) \sim 0.5$. This agrees with the value derived from the IUE spectra (see Sect. 2.1). Thus we feel confident that the procedure we applied above gives reliable results. From the fits we derived the column densities listed in Table 2. We have previously obtained the oxygen abundance of $\mathrm{H}$ II regions in Haro $11, \log (N(\mathrm{O}) / N(\mathrm{H}))+12=7.9$. We will assume that this is an upper limit of the oxygen abundance in the neutral region. Applying the conversion to hydrogen column densities from this number we obtain a lower limit to the hydrogen column density, $\log N(\mathrm{H} \mathrm{I})=19.6$. We have no corresponding determination of the remaining elements but if we assume the relative abundances to be the same as the solar (Asplund et al. 2004a) we obtain a lower $\operatorname{limit}$ to $\log N(\mathrm{H}$ I) of between 19.6 and 20.3 with the lower value for the strong carbon line. These relative high values are not consistent with a diffuse distribution of H I since it would prohibit leakage. We conclude that the photons must be leaking out through transparent windows in the gaseous halo in a "picket-fence fashion". This would also mean that the estimated column densities are slightly too low, due to the dilution. A possible scenario explaining why we observe a leakage is that the merging process has allowed young star clusters to separate from high density gaseous regions on a time scale shorter than the lifetime of the burst, i.e. in a short moment of strong gravitational disturbances.

In the following analysis we will assume that the picketfence model is the best approximation of the situation.

\subsection{Extinction corrections}

In the next section we will derive an estimate of the LyC escape fraction based on both the observed $\mathrm{H} \alpha$ flux and the continuum fluxes at 900 and $960 \AA$ rest wavelength of Haro 11, $f_{900}$ and $f_{960}$. The fluxes were integrated over spectral regions of widths 5 and $2 \AA$ respectively. As mentioned above, the $900 \AA$ continuum wavelength was chosen to allow a direct comparison with previous studies of Lyman leakage. The $960 \AA$ wavelength was chosen for spectral evolutionary model comparisons since it represents a spectral region reasonably close to the Lyman limit but unaffected by Lyman absorption line wings and other strong lines. The observed data need to be corrected for galactic and internal dust extinction. The galactic extinction in the direction of Haro 11 is $A_{B}=0.049$ (Schlegel et al. 1998). The 900 and $960 \AA$ rest wavelengths are redshifted to $\sim 920$ and $980 \AA$ in the observed spectrum. At these wavelength $A_{B}=0.049$ corresponds to $A_{920}=0.177$ and $A_{980}=0.175$ (Mathis 1990). We will now discuss the internal extinction corrections.

In the picket-fence approximation, as is further detailed in the next section, we will assume that the LyC photons are escaping through dust free windows. The only goal for the remaining part of this section is thus to obtain a value of the extinction correction to the $960 \AA$ region, $A_{960}$. From the $\mathrm{H} \alpha / \mathrm{H} \beta$ ratio of the central $4^{\prime \prime} \times 4^{\prime \prime}$, corrected for underlying absorption (Bergvall \& Östlin 2002), we obtain, assuming the Calzetti et al. (1994) extinction law, an internal extinction in $B$ of $A_{B}=1.08^{\mathrm{m}} \pm 0.07^{\mathrm{m}}$. The FUSE aperture is $30^{\prime \prime} \times 30^{\prime \prime}$, i.e. much larger than the aperture of the optical spectroscopy. The flux inside the $4^{\prime \prime} \times 4^{\prime \prime}$ aperture is only about $10 \%$ of the total flux so an estimate of the global extinction based on these data is quite uncertain. If we assume that the dust column density is 
proportional to the $B$ band flux we use the small aperture data to derive total extinction in $B$ to be $A_{B}=0.66^{\mathrm{m}} \pm 0.08^{\mathrm{m}}$. The error does not include the uncertainty of the model of the dust distribution.

We can also estimate the extinction from the observed slope of the UV continuum in Haro 11, using a method developed by Calzetti et al. (1994, 1995) and Meurer et al. (1995). Calzetti et al. (1994) investigated 39 starburst and blue compact galaxies to study the interstellar extinction in the UV. The observed spectral distribution in this region may be approximated by a power law $F(\lambda) \propto \lambda^{\beta}$. They found that the optical depth obtained from the $\mathrm{H} \alpha / \mathrm{H} \beta$ ratio correlated with the slope $\beta$ and derived a value of $\beta$ in a dust free environment, $\beta_{0}=-1.7$. They also found that the optical depth calculated from the $\mathrm{H} \alpha / \mathrm{H} \beta$ ratio was a factor of 2 larger than that derived from the continuum underlying the emission lines. This was interpreted as a consequence of a systematically higher extinction in young star forming regions as compared to older regions.

Later, Meurer et al. (1995) found a correlation between $\beta$ and the ratio between the far infrared flux and the UV flux at $2200 \AA$ A . This correlation agreed very nicely with a foreground dust screen model following the Calzetti et al. extinction law, if the galaxies had a similar intrinsic UV continuum distribution. The observed slope can therefore be used to derive an estimate of the extinction by comparing to the "standard" dust free slope. The value derived by Meurer et al. $\beta_{0}=-2.5 \pm 0.5$, is bluer than what Calzetti et al. found. This is probably due to the fact that the Meurer et al. sample contained more lowluminosity starburst dwarfs that were completely dominated by the young population while the more massive galaxies in the Calzetti et al. sample had a slightly larger contribution from older stars. The extinction of Haro 11 therefore depends on which value we choose. Haro 11 is involved in a very intense global starburst and is metal poor. On the other hand it is quite luminous and most likely has quite a complex star formation history so we expect a mixture of stellar populations of different ages. This puts Haro 11 somewhere in between the Meurer et al. sample and the Calzetti et al. sample. We will therefore assume that its dust free UV continuum is represented by the weighted mean of the Meurer et al. value and the Calzetti et al. value. Both have a similar observed scatter around the mean so the value we will adopt is $\beta_{0}=-2.1 \pm 0.2$.

The UV slope in Haro 11, obtained from our IUE observations, covering the wavelength interval $1250-1980 \AA$ is $\beta=-1.4 \pm 0.1$. (In the picket-fence model it is incorrect to use the slope without correcting for the contribution from the unobscured part, but this would make a negligible difference here.) As an additional check of this value we can use the observed flux ratio $F_{\mathrm{FIR}} / F_{\mathrm{UV}}$ of Haro 11 and apply the model most favoured by Meurer et al. to calculate the predicted value of $\beta . F_{\mathrm{FIR}}$ is derived from IRAS data and the approximation from Lonsdale \& Helou (1985). This results in $F_{\mathrm{FIR}}=$ $1.26 \times 10^{-14}\left(2.58 F_{60}+F_{100}\right) \mathrm{W} \mathrm{m}^{-2}=2.74 \times 10^{-13} \mathrm{~W} \mathrm{~m}^{-2}$. The flux at $2200 \AA$ was obtained from an extrapolation of the fit to the shorter wavelength region. This gives us $F_{\mathrm{FIR}} / F_{\mathrm{UV}}=5.9$. From this value we obtain $\beta=-1.6 \pm 0.1$, agreeing nicely with the former value we derived. $\beta=-1.4$ corresponds to an extinction in $B$ of $A_{B}=0.83$, which is quite close to the value we obtain from the $\mathrm{H} \alpha / \mathrm{H} \beta$ ratio $\left(A_{B}=0.66\right)$. We will assume a value of $A_{B}=0.7 \pm 0.2$.

The final value of the internal extinction was obtained in two steps. First we adopted the Calzetti et al. extinction law, relevant in the wavelength region $1250-8000 \AA$, to determine the reddening correction at $1250 \AA$. Then we used the recent determination of the extinction curve in the SMC by Cartledge et al. (2005) to go from there to $960 \AA$, the region we will use to sample the stellar continuum. We thus finally adopt an internal extinction $A_{960}=2.9^{\mathrm{m}} \pm 0.3$.

We will now, with the use of spectral evolutionary models, derive an estimate of the upper and lower flux escape limits.

\subsection{Estimate of the escape fraction}

\subsubsection{Comparison with spectral evolutionary models}

Under the assumption of a simple geometry for the distribution of stars, gas and dust, limits on the global LyC escape fraction from Haro 11 may be inferred from the observed LyC flux. In principle, the LyC leakage could be caused either by holes in the gas (e.g. supernova chimneys), by a total gas mass too low to form a complete Strömgren sphere, or by a combination thereof. Since the high column densities of neutral hydrogen derived in Sect. 3.1 disfavour a truncated Strömgren sphere for Haro 11, we will here estimate the escape fraction assuming that the escape is exclusively due to an isotropic distribution of holes, which are assumed to be devoid of gas (neutral and ionized) as well as dust.

Following Deharveng et al. (2001), we define the total LyC escape fraction $f_{\text {esc }}$ as

$f_{\text {esc }}=\frac{L_{900, \mathrm{obs}}}{L_{900, \star}}$

where $L_{900, o b s}$ represents the observed (escaping) luminosity (W $\AA^{-1}$ ) at a wavelength corresponding to $900 \AA$ in the rest system of the galaxy, and $L_{900, \star}$ represents the corresponding luminosity produced by the stellar component before attenuation by gas. Under the approximations adopted here, $L_{900, \star}$ is given by the sum of two components:

$L_{900, \star}=L_{900, \mathrm{obs}}+L_{900, \mathrm{abs}}$,

where $L_{900, a b s}$ represents the luminosity lost through gas absorption inside the galaxy. $L_{900, \text { abs }}$ is in turn related to the number of LyC photons absorbed by gas $N_{\text {LyC,abs }}$ through

$L_{900, \mathrm{abs}}=N_{\mathrm{LyC}, \mathrm{abs}} \times 10^{-k_{1}}$,

where $k_{1}$ is a model-dependent parameter. Under the assumption of case B combination, the number of absorbed LyC photons can then be related to the extinction-corrected $\mathrm{H} \alpha$ luminosity $L_{\mathrm{H} \alpha}$ through

$N_{\mathrm{LyC}, \mathrm{abs}}=\frac{\lambda_{\mathrm{H} \beta}}{h c\left(j_{\mathrm{H} \alpha} / j_{\mathrm{H} \beta}\right)} \frac{\alpha_{\mathrm{B}}}{\alpha_{\mathrm{H} \beta}^{\mathrm{eff}}} L_{\mathrm{H} \alpha}$,

where $j_{\mathrm{H} \alpha} / j_{\mathrm{H} \beta}$ is the intrinsic $\mathrm{H} \alpha / \mathrm{H} \beta$ line ratio, $\alpha_{\mathrm{B}}$ is the Case B recombination coefficient and $\alpha_{\mathrm{H} \beta}^{\mathrm{eff}}$ is the effective recombination coefficient for the $\mathrm{H} \beta$ line. These three parameters 
Table 3. The grid of Z01 evolutionary sequences. The grid consists of all possible combinations of the parameter values listed below. Each evolutionary sequence runs from ages of 0 to $100 \mathrm{Myr}$ in short time steps.

\begin{tabular}{ll}
\hline \hline IMF, $\alpha$ & $1.35,2.35,3.35$ \\
$M_{\text {up }}$ & $20,70,120 M_{\odot}$ \\
SFH & e $10, \mathrm{c} 100$ \\
$Z_{\star}$ & 0.001 \\
\hline
\end{tabular}

IMF: $\mathrm{d} N / \mathrm{d} M \propto M^{-\alpha}$.

$M_{\text {up }}$ : the upper mass limit of the IMF.

$\mathrm{SFH}=$ Star formation history.

$\mathrm{c}=$ Constant star formation rate during the subsequent number of Myr. $\mathrm{e}=$ Exponentially declining star formation rate, $S F R(t) \propto \exp (-t / \tau)$, with an e-folding decay rate $(\tau)$ equal to the subsequent number of Myr.

$Z_{\star}$ : the metallicity of the stellar population.

depend on the temperature of the nebula and, to lesser extent, on the density. By assuming an electron density of $100 \mathrm{~cm}^{-3}$ and fitting a cubic spline to the $j_{\mathrm{H} \alpha} / j_{\mathrm{H} \beta}, \alpha_{\mathrm{B}}$ and $\alpha_{\mathrm{H} \beta}^{\text {eff }}$ values given by Osterbrock (1989), we estimate that for the electron temperature of Haro $11(T \approx 13400 \mathrm{~K}$; Bergvall \& Östlin 2002):

$N_{\text {LyC,abs }} \approx 8.56 \times 10^{11} L_{\mathrm{H} \alpha}$.

Combination of the equations above, with the relation $L=$ $4 \pi D^{2} f$ between luminosity $L, \mathrm{H} \alpha$ flux $f_{\mathrm{H} \alpha}$, flux density $f_{\lambda}$ and distance $D$, gives:

$f_{\mathrm{esc}}=\frac{f_{900, \mathrm{obs}}}{f_{900, \mathrm{obs}}+8.56 \times 10^{11-k_{1}} f_{\mathrm{H} \alpha}}$.

Constraints on $f_{\text {esc }}$ may be derived from (8) by combining the estimated $1 \sigma$ error bars on $f_{900, \text { obs }}$ and $f_{\mathrm{H} \alpha}$ with extremum values of $k_{1}$ derived from models. When evaluating the plausible range of the $k_{1}$ parameter, defined as

$k_{1}=\log \left(\frac{N_{\text {LyC,abs }}}{L_{900, \text { abs }}}\right)$,

we use the Zackrisson et al. (2001, hereafter Z01) spectral evolutionary model. With this model, a grid of burst-like spectral evolutionary sequences is generated, defined by the model parameter values specified in in Table 3 . In the case of a standard Salpeter IMF $\left(\mathrm{d} N / \mathrm{d} M \propto M^{-\alpha} ; \alpha=2.35\right.$ with upper mass limit $\left.M_{\text {up }}=120 M_{\odot}\right)$ the range of $k_{1}$ values allowed by this grid of star formation scenarios becomes $k_{1}=13.23-13.24$. When the full range of IMF variations considered ( $\alpha=1.35-3.35$ and $M_{\text {up }}=20-120 M_{\odot}$ ) is used, this constraint relaxes to $k_{1}=12.70-13.58$.

When these values of $k_{1}$ are combined with the previously discussed measurements of $f_{900, \text { obs }}=1.06 \pm 0.09 \times$ $10^{-17} \mathrm{~W} \mathrm{~m}^{-2} \AA^{-1}$ and $f_{\mathrm{H} \alpha}=4.5 \pm 0.5 \times 10^{-15} \mathrm{~W} \mathrm{~m}^{-2}$, limits on $f_{\text {esc }}$ may be inferred, resulting in $0.04 \leq f_{\text {esc }} \leq 0.10$ for the standard IMF and $0.01 \leq f_{\text {esc }} \leq 0.11$ for the more generous range of IMF scenarios.

These $f_{\text {esc }}$ estimates rely heavily on the conversion from $L_{\mathrm{H} \alpha}$ to $L_{900, \text { abs }}$, and are only valid under the assumption that the
$\mathrm{H} \alpha$ line is dominated by photoionization by stars, i.e. that ionization contribution from shocks or an active galactic nucleus are negligible. We have also assumed the dust to be exclusively located outside the Strömgren sphere, so that no LyC photons are lost due to internal dust extinction. Potential far-UV opacity sources outside Haro 11, other than dust in the Milky Way, have furthermore been neglected.

A consistency test may be carried out by comparing the observed slope of the spectrum across the Lyman limit, to the corresponding slope predicted by models. To do this, we measure the continuum flux density at a rest wavelength of $960 \AA$, where the spectrum should be completely unaffected by gaseous absorption. After correction for Galactic extinction, the observed flux density at this wavelength is $f_{960, \mathrm{obs}}=$ $7.4 \pm 0.4 \times 10^{-17} \mathrm{~W} \mathrm{~m}^{-2} \AA^{-1}$. In Sect. 3.2 the correction factor for internal extinction was estimated to be $y_{960}=14_{-3}^{+5}$. Given the assumption that the holes in the nebula are completely devoid of both gas and dust, and hence that the dust correction applies only to the parts of the nebula where there is no LyC leakage, the escape fraction may then be estimated from:

$f_{\text {esc }}=\frac{k_{2} f_{900, \mathrm{obs}}}{y_{960} f_{960, \mathrm{obs}}-\left(y_{960}-1\right) k_{2} f_{900, \mathrm{obs}}}$,

where $k_{2}$ represents the ratio of the stellar component fluxes at $960 \AA$ to $900 \AA$ prior to absorption by gas and dust:

$k_{2}=\frac{f_{960, \star}}{f_{900, \star}}$

Although the exact value of $k_{2}$ is not known for Haro 11, a lower limit on $f_{\text {esc }}$ can be imposed from the minimum $k_{2}$ predicted by the plausible range of star formation scenarios. From the model grid defined in Table 3, we infer $\min \left(k_{2}\right)=1.78$ for the standard IMF and $\min \left(k_{2}\right)=1.42$ otherwise. This translates into $f_{\mathrm{esc}} \geq 0.025_{-0.010}^{+0.012}$ and $f_{\mathrm{esc}} \geq 0.019_{-0.003}^{+0.009}$, respectively, which is completely consistent with the LyC escape fraction derived from the $\mathrm{H} \alpha$ flux.

To further test the robustness of these results, two other spectral evolutionary models - Starburst99 v. 4 (Leitherer et al. 1999) and PÉGASE.2 (Fioc \& Rocca-Volmerange 1999) have been used to assess the model-sensitivity of the $k_{1}$ and $k_{2}$ parameters. We find, that when identical input parameters are used in all codes, very similar results are produced, indicating that our limits on $f_{\text {esc }}$ do not critically depend on the use of any specific model.

\section{Discussion}

As we mentioned in the introduction it has been argued that star-forming galaxies may yield the required LyC emissivities to reionize the universe at $z>5-6$. This approach has the advantage over assuming AGNs as sources in that it explains the early pollution of heavy elements observed in the IGM at these redshifts (e.g. Cowie et al. 1995). Moreover, analysis of the optical depth at the H I and HeII Ly $\alpha$ continuum breaks in different environments at $z<4$ indicates that the major ionization source in dense regions is QSOs while in void regions the radiation appears to be softer, giving room for a substantial contribution from starburst galaxies. 
Several semi-analytical methods have been used to model the reionization epoch. Due to the strong inhomogeneities of the radiation field in the early stages of the reionization, the computations are however quite demanding and complex (see Razoumov et al. 2002 for a summary of the technical problems) and realistic simulations have not been possible until recently. In 2003, Sokasian et al. (2003) used a fast code capable of exploring the impact of low-mass starburst galaxies in high spatial resolution. They focused on the evolution of the interaction between the IGM and starforming galaxies of normal properties, e.g. similar to Haro 11, in the redshift interval $z \sim 20$ to $z \sim 6$. One of the results was that the previous estimates of the required photon flux rate needed to complete the reionization was overestimated by $63 \%$ if assumed that only galaxies with masses $>10^{9} h^{-1} \mathcal{M}_{\odot}$ would account for the reionization. By including sources with masses down to $\sim 4 \times 10^{7} \mathrm{~h}^{-1} \mathcal{M}_{\odot}$ the impact of the large population of dwarf starburst galaxies could be realistically assessed. The calculations demonstrated that the soft ionization sources of type starburst dwarfs give the required photon flux provided that the escape fraction is $f_{\text {esc }} \geq 0.20$. This number is twice the upper limit of $f_{\text {esc,gas }}$ that we derived for Haro 11. The estimate is however based on a normal stellar population. At redshifts $z>10$ pop III stars could dominate the population (e.g. Choudhury \& Ferrara 2005). In these stars the emissivity in the UV might be larger than in pop II by a factor of 2 (Tumlinson \& Shull 2000; Bromm et al. 2001). Thus, although $f_{\text {esc }}$ would not change, the number of produced photons per unit mass would increase significantly. Thus it seems feasible that, at least at $z \gtrsim 10$, dwarf starbursts of the mass of Haro 11 and lower could provide a substantial contribution to the early phase of the reionization process.

\section{Conclusions}

We report on the discovery of a weak signal in the Lyman continuum of the luminous starburst galaxy Haro 11. It is the first time Lyman continuum leakage is found in a galaxy in the local environment. Using the derived column densities of low ionization species we estimate the column density of neutral hydrogen to be $\sim 10^{19} \mathrm{~cm}^{-2}$, which is too high to allow Lyman continuum photon escape if the gas is diffusely distributed. We conclude that the Lyman continuum radiation is escaping through transparent windows of the ISM. Assuming a Salpeter IMF, we estimate a total escape fraction of $0.04<f_{\text {esc }}<0.10$. More extreme IMFs allow a lower escape fraction but the major uncertainty is due to the problematic background subtraction at short wavelengths. The upper limit is below the model estimates of the minimum escape rate required for reionization. Still, this single case demonstrates that a significant fraction of the required Lyman continuum photons may be produced by starburst dwarfs, in particular if we also consider that Pop III stars, that should dominate the first generation galaxies, are more efficient than Pop II stars in producing Lyman continuum photons.

Acknowledgements. This work has been done using the profile fitting procedure Owens.f developed by M. Lemoine and the FUSE French
Team. We are indebted to Carmelle Robert for help with the access to the LavalSB synthetic spectral library. We are pleased to thank our referee, Daniel Kunth, for many valuable comments and suggestions to improvements of the manuscript. Kjell Olofsson is thanked for his observational contribution to the IUE data. This work was supported by the Swedish Space Board and Swedish Research Council.

\section{References}

Asplund, M., Grevesse, N., \& Sauval, A. J. 2004a, in Cosmic abundances as records of stellar evolution and nucleosynthesis, ed. F. N. Bash, \& T. G. Barnes, ASP Conf. Ser., in press

Asplund, M., Grevesse, N., Sauval, A. J., Allende Prieto, C., \& Kiselman, D. 2004b, A\&A, 417, 751

Becker, R. H., Fan, X., White, R. L., et al. 2001, AJ, 122, 2850

Begum, A., \& Chengalur, J. N. 2005, MNRAS, 362, 609

Bergvall, N. 1985, A\&A, 146, 269

Bergvall, N., \& Olofsson, K. 1986, A\&AS, 64, 469

Bergvall, N., Masegosa, J., Östlin, G., \& Cernicharo, J. 2000, A\&A, 359,41

Bergvall, N., \& Östlin, G. 2002, A\&A, 390, 891

Bresolin, F., Kennicutt, R. C., \& Garnett, D. R. 1999, ApJ, 510, 104

Bromm, V., Kudritzki, R. P., \& Loeb, A. 2001, ApJ, 552, 464

Burton, W. B., \& Hartmann, D. 1994, Ap\&SS, 217, 189

Calzetti, D., Kinney, A. L., \& Storchi-Bergmann, T. 1994, ApJ, 429, 582

Calzetti, D., Bohlin, R. C., Kinney, A. L., et al. 1995, ApJ, 443, 136

Cartledge, S. I. B., Clayton, G. C., Gordon, K. D., et al. 2005, AJ, 630, 355

Choudhury, T. R., \& Ferrara, A. 2005, MNRAS, 361, 577

Cowie, L., Songaila, A., Kim, T., \& Hu, E. M. 1995, AJ, 109, 1522

Deharveng, J.-M., Buat, V., Le Brun, V., et al. 2001, A\&A, 375, 805

Devriendt, J. E. G., Sethi, S. K., Guiderdoni, B., \& Nath, B. B. 1998, MNRAS, 298, 708

Fan, X., Narayanan, V. K., Lupton, R. H., et al. 2001, AJ, 122, 2833

Fan, X., Narayanan, V. K., Strauss, M. A., et al. 2002, AJ, 123, 1247

Feldman, P. D., Sahnow, D. J., Kruk, J. W., Murphy, E. M., \& Moos, H. W. 2001, JGR, 106, 8119

Ferguson, A. M. N., Wyse, R. F. G., Gallagher, J. S., III, \& Hunter, D. A. 1996, AJ, 111, 2265

Fernandez-Soto, A., Lanzetta, K. M., \& Chen, H.-W. 2003, MNRAS, 342, 1215

Fioc, M., \& Rocca-Volmerange, B. 1999 [arXiv: astro-ph/9912179]

Giallongo, E., Fontana, A., \& Madau, P. 1997, MNRAS, 289, 629

Gonzalez Delgado, R. M., Leitherer, C., \& Heckman, T. 1997, ApJ, 489, 601

Gordon, D., \& Gottesman, S. T. 1981, AJ, 86, 161

Guzmán, R., Östlin, G., Kunth, D., et al. 2003, ApJ, 586, L45

Heckman, T. M., Sembach, K. R., Meurer, G. R., et al. 2001, ApJ, 558, 56

Hirashita, H., Buat, V., \& Inoue, A. K. 2003, A\&A, 410, 83

Hurwitz, M., Jelinsky, P., \& Dixon, W. V. D. 1997, ApJ, 481, L31

Iglesias-Páramo, J., \& Muñoz-Tuñón, C. 2002, MNRAS, 336, 33

Inoue, A. K., Iwata, I., Deharveng, J.-M., Buat, V., \& Burgarella, D. 2005, A\&A, 435, 471

Jenkins, E. B., Oegerle, W. R., Gry, C., et al. 2000, ApJ, 538, L81

Kitayama, T., Yoshida, N., Susa, H., \& Umemura, M. 2004, ApJ, 613, 631

Kogut, A., Spergel, D. N., Barnes, C., et al. 2003, ApJS, 148, 161

Kunth, D., Mas-Hesse, J. M., Terlevich, E., et al. 1998, A\&A, 334, 11

Lehner, N. 2002, ApJ, 578, 126 
Leitherer, C., Ferguson, H. C., Heckman, T. M., \& Lowenthal, J. D. 1995, ApJ, 454, L19

Leitherer, C., Vacca, W. D., Conti, P. S., et al. 1996, ApJ, 465, 717

Leitherer, C., Schaerer, D., Goldader, J. D., et al. 1999, ApJS, 123, 3

Lilly, S., Schade, D., Ellis, R., et al. 1998, ApJ, 500, 75

Lonsdale, C. J., \& Helou, G. 1985, Catalogued galaxies and quasars observed in the IRAS survey, Pasadena: JPL

Madau, P., Haardt, F., \& Rees, M. J. 1999, ApJ, 514, 648

Madau, P., Rees, M. J., Volonteri, M., Haardt, F., \& Oh, S. P. 2004, ApJ, 604, 484

Mallén-Ornelas, G., Lilly, S. J., Crampton, D., \& Schade, D. 1999, ApJ, 518, L83

Mas-Hesse, J. M., \& Kunth, D. 1999, A\&A, 349, 765

Mathis, J. S. 1990, ARA\&A, 28, 37

Meiksin, A., \& White, M. 2003, MNRAS, 342, 1205

Mesinger, A., \& Haiman, Z. 2004, ApJ, 611, 69

Meurer, G. R., Heckman, T. M., Leitherer, C., et al. 1995, AJ, 110, 2665

Miller, J. S., \& Mathews, W. G. 1972, ApJ, 172, 593

Moy, E., Rocca-Volmerange, B., \& Fioc, M. 2001, A\&A, 365, 347

Oey, M. S., \& Kennicutt, R. C., Jr. 1997, MNRAS, 291, 827

Östlin, G., Amram, P., Masegosa, J., Bergvall, N., \& Boulesteix, J. 1999, A\&AS, 137, 419

Östlin, G., Amram, P., Bergvall, N., Masegosa, J., \& Boulesteix, J. 2001, A\&A, 374, 800
Östlin, et al. 2005, in preparation

Osterbrock, D. E. 1989, Astrophysics of gaseous nebulae and active galactic nuclei (University Science Books)

Razoumov, A. O., Norman, M. L., Abel, T., \& Scott, D. 2002, ApJ, 572, 695

Robert, C., Pellerin, A., Aloisi, A., et al. 2003, ApJS, 144, 21

Savage, B. D., \& Sembach, K. R. 1991, ApJ, 379, 245

Schlegel, D. J., Finkbeiner, D. P., \& Davis, M. 1998, ApJ, 500, 525

Seaton, M. J. 1979, MNRAS, 187, 73

Sembach, K. R., Howk, J. C., Savage, B. D., \& Shull, J. M. 2001, AJ, 121, 992

Shull, J. M., Roberts, D., Giroux, M. L., Penton, S. V., \& Fardal, M. A. 1999, AJ, 118, 1450

Sokasian, A., Abel, T., Hernquist, L., \& Springel, V. 2003, MNRAS, 344,607

Stasińska, G. 1990, A\&AS, 83, 501

Stasińska, G., Schaerer, D., \& Leitherer, C. 2001, A\&A, 370, 1

Steidel, C. C., Pettini, M., \& Adelberger, K. L. 2001, ApJ, 546, 665

Tumlinson, J., \& Shull, J. M. 2000, ApJ, 528, L65

Yan, H., \& Windhorst, R. A. 2004, ApJ, 600, L1

Wood, K., \& Mathis, J. S. 2004, MNRAS, 353, 1126

Zackrisson, E., Bergvall, N., Olofsson, K., \& Siebert, A. 2001, A\&A, $375,814(\mathrm{Z} 01)$

Zurita, A., Beckman, J. E., Rozas, M., \& Ryder, S. 2002, A\&A, 386, 801 